\title{
Assistência de enfermagem a gestante com hipertensão na Atenção Básica: um relato de experiência
}

\author{
Nursing assistance to pregnant women with hypertension in Basic Care: na experience report \\ Assistencia de enfermeira a mujeres embarazadas com hipertensión em Cuidados Básicos: um \\ relato de experiência
}

Pâmela Souza Bastos

ORCID: https://orcid.org/0000-0002-7886-8331

Faculdade Irecê, Brasil

E-mail: pamelajp.1024@hotmail.com

Kelle Karolina Ariane Ferreira Alves

ORCID: https://orcid.org/0000-0002-1187-0429

Faculdade Irecê, Brasil

E-mail: Kellekarolina@hotmail.com

Flávia Gomes Silva

ORCID: https://orcid.org/0000-0002-6505-2077 Instituto Federal da Paraíba, Brasil E-mail: flavianag12@gmail.com

Wesley Dias Pereira

ORCID: https://orcid.org/0000-0003-2686-1020 Universidade Gunambi, Brasil

E-mail: W_diaspereira@hotmail.com

Cintia Ferreira Amorim

ORCID: https://orcid.org/0000-0002-4650-8278 Faculdade Irecê, Brasil E-mail: cicis2006@hotmail.com

Carine Barreto de Carvalho

ORCID: https://orcid.org/0000-0003-3579-9521 Faculdade Irecê, Brasil E-mail: cbarreto21.cb@gmail.com

Cleuton Machado Cavalcante ORCID: https://orcid.org/0000-0001-9903-1285 Faculdade Irecê, Brasil

E-mail: cleuton.saudecoletiva@gmail.com

Nádia Shirlley de Andrade Cavalcante ORCID: https://orcid.org/0000-0001-8200-3691 Faculdade Irecê, Brasil

E-mail: nadja.gestaoeducacional@gmail.com

Luana Queiroz Oliveira

ORCID: https://orcid.org/0000-0002-6326-8579 Faculdade Irecê, Brasi

E-mail: luua.enf@outlook.com

\begin{abstract}
Resumo
Objetivo: descrever sobre a assistência de enfermagem à gestante com hipertensão na atenção básica. Métodos: Tratase de um estudo de caráter descritivo e abordagem qualitativa na modalidade relato de experiência. Sendo este, vivenciado por uma discente do curso de Bacharelado em enfermagem, durante o estágio supervisionado I, realizado em UBSF de uma cidade situada no interior da Bahia. Resultados: Durante as consultas de pré-natal as gestantes com diagnóstico de SHG caracterizou-se como um grande desafio, e de grande valia para formação, sendo este oportuno para o desenvolvimento como futuro profissional, o qual trouxe muita aprendizagem. $\mathrm{O}$ enfermeiro e médico que realizam o pré-natal de baixo risco devem estar vigilantes quanto ao rastreamento e diagnóstico, com isso torna-se imprescindível a anamnese, exame físico, ginecológico quando necessário, e obstétrico, além da promoção de atividades educativas. Conclusão: É evidente que ainda existem algumas lacunas relacionadas ao diagnóstico precoce das SHG, o que na maioria das vezes é consequência do início tardio do pré-natal.
\end{abstract}

Palavras-chave: Hipertensão induzida pela gravidez; Complicações na gravidez; Eclampsia.

\section{Abstract}

Objective: to describe nursing care for pregnant women with hypertension in primary care. Methods: This is a descriptive study with a qualitative approach in the experience report modality. This being experienced by a student of 
the Bachelor of Nursing course, during supervised internship I, held at UBSF in a city located in the interior of Bahia. Results: During prenatal consultations, pregnant women diagnosed with GHS were characterized as a great challenge, and of great value for training, which is opportune for the development as a professional future, which brought a lot of learning. Thus, it is worth mentioning that the nurse must be vigilant as to the risk classification in all consultations with pregnant women, thus anamnesis, general physical, gynecological, and obstetric examination, in addition to the promotion of educational activities, are essential. Conclusion: It is evident that there are still some gaps related to the early diagnosis of GHS, which in most cases is a consequence of the late start of prenatal care.

Keywords: Pregnancy-induced hypertension; Pregnancy complications; Eclampsia.

\section{Resumen}

Objetivo: describirlaatención de enfermería a gestantes conhipertensión arterial enatención primaria. Métodos: Se trata de unestudiodescriptivocon enfoque cualitativoenlamodalidad de relato de experiencia. Estolo vive una estudiantedel curso de Licenciatura enEnfermería, durante lapasantía supervisada I, realizada enla UBSF en una ciudadubicadaenel interior de Bahía. Resultados: Durante las consultas prenatales, las gestantes diagnosticadas con GHS se caracterizaron como ungrandesafío, y de gran valor para laformación, que es oportuna para eldesarrollo como futuro profesional, que trajo muchoaprendizaje. Así, cabe mencionar que elenfermerodebe estar atento a laclasificación de riesgoen todas las consultas con gestantes, por lo que laanamnesis, laexploración física general, ginecológica y obstétrica, además de lapromoción de actividades educativas, sonfundamentales. Conclusión: Es evidente que aún existen algunas brechas relacionadas conel diagnóstico precozdel SGA, que en la mayoría de los casos es consecuencia del inicio tardio de la atención prenatal.

Palabras clave: Hipertensióninducida por el embarazo; Complicaciones del embarazo; Eclampsia.

\section{Introdução}

A Hipertensão arterial sistêmica (HAS) é considerada como a patologia que causa complicações na gravidez com maior frequência, além de ser uma das principais causas de morbimortalidade materna e perinatal, visto que o diagnóstico precoce e o tratamento adequado são de fundamental importância para melhorar os resultados maternos e perinatais, já que a gravidez pode impulsionar a HAS em mulheres normotensas ou até mesmo agravar uma hipertensão preexistente (Brasil, 2012).

Conforme a Febrasgo (2017) algumas evidências descritas na literatura, retratam que essa patologia gestacional pode estar interligada ao sistema imunológico materno, devido ao processo de adaptação imunológica, considerados ainda como gatilhos para uma série de alterações no organismo, como distúrbios na perfusão celular, podendo evoluir para uma hipóxia celular.

Atrelado a esse processo, o diagnóstico de HAS na gravidez é realizado quando os níveis pressóricos são iguais ou superior a 140/90 mmHg, consideradas como Síndrome Hipertensiva da Gestação (SHG), o qual pode ser classificada em: préeclâmpsia; eclampsia (doença hipertensiva específica da gravidez); hipertensão crônica; pré-eclâmpsia sobreposta à hipertensão crônica; hipertensão gestacional (Moura Marta, Castro, Margotto \& Rugolo, 2011).

Diante disso o Ministério da Saúde Brasil (2012), destaca que o diagnóstico diferencial pode se tornar difícil quando a gestante comparece a primeira consulta após a $20^{\mathrm{a}}$ semana com HAS e não sabem informar seus níveis pressóricos anteriores. Tendo em vista que, quando a gestante é primigesta e continua com seu parceiro de sua primeira gestação, e se tiver ácido úrico sérico menor que 4,5 mg/dl, o diagnóstico de HAS crônica é mais provável, e pré-eclâmpsia sobreposta a HAS crônica quando houver piora dos níveis pressóricos.

Dessa forma, sabe-se que existem alguns fatores de risco descritos na literatura para a SHG, tais como: condições socioeconômicas e demográficas desfavoráveis, como baixa escolaridade e baixa renda familiar, têm sido destaques relacionados ao surgimento desses agravos, levando as mulheres a uma gestação de risco, geralmente interligadas a piores condições nutricionais e obstétricas (Oliveira \& Graciliano, 2015).

Dado isso, o enfermeiro como um dos protagonistas principais frente às consultas de pré-natal a gestante que também é acompanhada no pré-natal de alto risco pelo obstetra, tendo em vista que é de relevância avaliação dos níveis pressóricos, e 
estado nutricional junto aos exames, para uma conduta qualificada, sendo essa embasada pelos protocolos fornecidos pelo Ministério da Saúde (MS) (Brasil, 2012).

Neste contexto, o questionamento que direcionou está pesquisa foi: quais são as evidências disponíveis na literatura sobre hipertensão arterial na gestação, e com isso despertou-se, o interesse em realizar essa pesquisa a partir da experiência adquirida como graduando de enfermagem durante as práticas do estágio curricular na Unidade Básica de Saúde da Família (UBSF), mediante ao atendimento de enfermagem prestado a gestante com SHG durante as consultas de pré-natal. Desta forma, objetiva-se descrever sobre a assistência de enfermagem a gestante com hipertensão na atenção básica sob a ótica de um discente de enfermagem.

\section{Metodologia}

Trata-se, de um estudo de caráter descritivo e abordagem qualitativa na modalidade relato de experiência, visto que o relato de experiência é considerado um tipo de estudo metodológico que permite descrever experiências vivenciadas pelo autor (Gil, 2010). Sendo este, vivenciado por uma discente do curso de Bacharelado em enfermagem, durante o estágio supervisionado I, realizado em UBSF de uma cidade situada no interior da Bahia.

Discente do décimo período do curso bacharelado de enfermagem se configurou como sujeito desse relato, detalhando sua vivência no estágio supervisionado I, com gestantes diagnosticadas com SHG durante as consultas de pré-natal sendo elas acompanhadas conforme é preconizado pelo MS de acordo com a idade gestacional. A experiência ocorreu entre os meses de setembro-dezembro do ano de 2020 de segunda a sexta-feira, sendo realizado consulta de enfermagem de todos os programas da UBSF (hiperdia, puericultura, saúde sexual e reprodutiva etc) sob a ótica de uma supervisora.

A população estimada de onde ocorreu a experiência, em 2020 de acordo o Instituto Brasileiro de Geografia e Estatística (IBGE) da cidade é de 18.789 pessoas, o qual é atendido na UBSF cerca de 3.600 pessoas, e a unidade não possui área descoberta, com um total de 32 gestantes, visto que a população que é atendida na UBSF é de baixa renda e dependem 100\% do Sistema Único de Saúde (SUS).

\section{Resultados}

No decorrer da vida acadêmica dentre as práticas desenvolvidas no atendimento, o discente tem a possibilidade de realizar o acompanhamento de pré-natal, com consultas de enfermagem às gestantes de baixo risco segundo protocolos do Ministério da Saúde na prática de campo da disciplina de Saúde da Mulher ou no Estágio Supervisionado. No estágio supervisionado I, tendo como diferencial do campo um total de 32 gestantes cadastradas durante o período, com aproximadamente 7 delas acompanhadas também no pré-natal de alto risco por motivos diversos, as estagiárias tiverem alguns desafios, no acompanhamento, solicitações de exames, avaliação de exames e orientações a essas gestantes.

O campo de estágio era rico, notou-se, que a maioria das gestantes vivia em situação de vulnerabilidade social tais como: pouco recurso financeiro, moradia precária, exposição às drogas e violência, o que pode ser considerado como fator desencadeante de uma gravidez de alto risco.

Outro destaque é em relação à idade dessas gestantes, algumas delas postergam a gravidez para depois dos 30 anos, ou de forma precoce no período da adolescência, além de histórico familiar de HAS e Diabetes Mellitus (DM) por parentes de primeiro grau, uso de drogas ou baixa adesão ao pré-natal. Todos esses fatores se apresentam como desafios para a assistência de enfermagem, e um diferencial na vida acadêmica das estagiárias, que ao vivenciarem essa realidade durante as consultas de enfermagem começam a refletir sobre as principais estratégias que devem ser adotadas para um enfrentamento mais efetivo.

Dentre as gestantes atendidas, algumas apresentavam apenas a Síndrome Hipertensiva Gestacional (SHG), enquanto outras foram posteriormente diagnosticadas também com a Diabetes Mellitus Gestacional (DMG). Durante a consulta realizada 
pelas estagiárias sob a supervisão da professora/supervisora, foi identificado em alguns casos a pressão arterial com o valor de 170x90 mmHg, associada a sintomas de cefaleia, epigastralgia, escotomas visuais, o que foi classificado no momento como pré-eclampsia sobreposta a hipertensão crônica, vale salientar que as mulheres estavam em uso de anti-hipertensivo previamente prescrito e foram encaminhadas para o pré-natal de alto risco e emergência. Além disso, receberam orientações durante as consultas de pré-natal sobre a relevância da alimentação balanceada, uso da medicação de forma correta como dose e horário, evitar estresse, ansiedade e sempre procurar ter um sono regular à noite, sendo realizado também o encaminhamento para a nutricionista.

Para as estagiárias realizarem um atendimento dessa complexidade, mesmo que com a supervisão e consulta compartilhada com a médica da unidade é um desafio, ademais retrata um momento de se apropriar da teoria vista em sala de aula, utilizando o raciocínio clínico. Diante desse cenário, viu-se a necessidade de uma abordagem multiprofissional, assim, ao final dos atendimentos eram realizadas reuniões entre a equipe da unidade e as estagiárias, para a discussão das condutas e estratégias de intervenções coletivas e individuais, sempre observando as evidências científicas.

Após uma dessas conversas viu-se a necessidade da elaboração de protocolo na unidade, foi montado um fluxograma com a definição, e condutas a serem realizadas com gestantes diagnosticadas com SHG, além de discussão produtiva entre os membros da equipe da UBSF e as estagiárias. Também foram confeccionados panfletos sobre a temática e distribuídos entre os setores da unidade (consultório de enfermagem, consultório médico, triagem, e sala de procedimentos).

Cabe ressaltar que o enfermeiro e o médico que realizam o pré-natal de baixo risco devem estar vigilantes quanto ao rastreamento e diagnóstico de DMG e SHG durante o acompanhamento do pré-natal. Além de realizarem classificação de risco em todos os atendimentos à gestante, com isso torna-se imprescindível a anamnese, exame físico, ginecológico quando necessário, e obstétrico, além da promoção de atividades educativas. Sabe-se também que o enfermeiro desempenha dentro da unidade diversas funções assistenciais e gerenciais, o que pode dificultar na assistência direta as usuárias gestantes que exigem mais tempo e cuidado.

Neste contexto, durante as consultas de pré-natal as gestantes com diagnóstico de SHG caracterizou-se como um grande desafio para a prática das acadêmicas, e de grande valia para formação, sendo este oportuno para o desenvolvimento das futuras profissionais. As experiências vivenciadas durante o estágio agregaram muita aprendizagem e reforçaram a importância da busca constante pelo conhecimento, para a prestação de um atendimento acolhedor e com qualidade para essas pacientes, além da importância do cuidado multidisciplinar.

\section{Discussões}

De igual modo Ferreira Maria, Silveira, Silva, Souza e Ruiz (2016), destacam que a paciente pode apresentar alguns indicativos de SHG, tais como: presença de edema em face, ao redor dos olhos e mãos, ganho acentuado de peso, náuseas/vômitos, dor em região epigástrica que irradia para os membros superiores, cefaléia, escotomas visuais e reflexos tendíneos, taquipneia, ansiedade, e às vezes a patologia pode evoluir de forma silenciosa. Atrelado a isso, a Organização Mundial de Saúde (OMS), preconiza o uso de protocolos assistenciais, no intuito de prevenção e tratamento.

A prevalência de mortalidade materna com SHG é de 60 a $80 \%$ e a fetal é de 56 a 75\%. Conforme um estudo realizado no Paraná, após ter feio a análise dos óbitos por SHG, notou-se que as maiorias dos casos poderiam ser evitadas com o diagnóstico precoce, e acompanhamento de uma equipe multidisciplinar em serviços especializados, sobretudo, a assistência ao pré-natal e puerpério de forma efetiva constitui-se como componente essencial e indispensável para redução da morbimortalidade materno-fetal (Kerber \& Melere, 2017).

Patologia essa, considerada como um problema importante de saúde pública e saúde da mulher, sendo mais comum em primigestas, multípara com idade avançada para a gravidez, gestante obesa, e aquelas com antecedentes familiares de 
hipertensão arterial, visto que, grávidas com essas condições apresentam maior risco de desenvolver diabetes gestacional e diabetes tipo 2. Estudos realizados na cidade Helsinquia, na Finlândia, e na Grécia mostraram que filhos de mães que tiveram complicações relacionadas à hipertensão na gravidez podem no futuro desenvolver deficiência cognitiva, problemas psiquiátricos e maior tendência a sofrer síndrome metabólica (Sousa Marida, Lopes, Rocha, Lippi, Costa \& Santos, 2020).

De acordo com Kerber e Melere (2017) e Vasconcelos Amorim, Souza, Moura, Queiroz e Salimena (2017) Sendo considerado como um distúrbio hipertensivo que mais causam complicações durante a gravidez. No que concerne sobre a HAS gestacional, vale enfatizar que essa doença continua sendo a principal causa de mortalidade materna, porém nos últimos anos essa taxa vem reduzindo, mesmo com sua etiologia desconhecida, nesse sentido destaca-se a importância do profissional enfermeiro no cuidado a gestante com SHG, e que quando aliado a uma equipe multiprofissional a assistência ocorre de forma dinâmica e resolutiva.

Em vista disso, o enfermeiro da UBSF tem como destaque priorizar a assistência de pré-natal no intuito de detectar risco de forma precoce. Dentre a dinâmica das consultas de pré-natal o enfermeiro deve estar vigilante quanto à classificação de risco em todos os atendimentos a gestante, com isso torna-se imprescindível a anamnese, exame físico geral, ginecológico, e obstétrico, além da promoção de atividades educativas, e assim atender seus anseios e necessidades particulares (Vasconcelos Amorim, et al., 2017).

Semelhante a isso, enfatizo sobre a relevância do estágio curricular na UBSF, o qual foi realizado atividades de grande destaque para o processo de graduação, visto que o campo nos proporcionou experiências marcantes, quanto a assistência de enfermagem a gestante com HAS, além de atendimento com demais públicos que ali são atendidos, nos programas ofertados pela atenção básica de saúde.

Desse modo, a SHG é uma patologia de grande repercussão na obstetrícia, requer cuidados específicos com a gestante e o concepto que está sendo gerado, dessa forma a assistência ao pré-natal é de grande importância para o diagnóstico precoce desta adversidade, e assim promover um cuidado qualificado e acolhedor dessas pacientes, já que a gravidez é uma das fases do ciclo da mulher que está relacionada a um misto de sensações de medo, alegria e anseios.

\section{Conclusão}

É evidente que ainda existem algumas lacunas relacionadas ao diagnóstico precoce das SHG, o qual na maioria das vezes é gestante que iniciam seu pré-natal de forma tardia, além da UBSF não realizar a busca ativa dessas gestantes, desse modo o diagnóstico precoce se torna algo desafiador para a equipe da unidade, que deve sempre buscar promover um acolhimento e uma ausculta qualificada dessas pacientes.

Levando-se em consideração o que foi mencionado anteriormente, esse estudo instiga o leitor a buscar conhecimento científico embasado nos protocolos do Ministério da Saúde de assistência a gestante com SHG, no intuito de promover atenção integral e humanizada as gestantes, e assim aprimorar seu conhecimento frente à patologia.

Fica evidente também a necessidade e implementação de estratégias preventivas com adoção de práticas saudáveis, mudança de estilo de vida e crenças propostas pelos enfermeiros de forma dinâmica, criativa alinhada com as necessidades da população adscrita ao serviço, sendo a prevenção o melhor método terapêutico.

Conclui-se que, o estágio supervisionado I contribuiu de forma significativamente para a construção profissional do envolvido, tendo em vista que durante a assistência prestada à população através do conhecimento científico adquirido ao longo da graduação mostrou de grande valia para toda a população do campo o qual ocorreu às atividades. 
Research, Society and Development, v. 10, n. 1, e55510112009, 2021

(CC BY 4.0) | ISSN 2525-3409 | DOI: http://dx.doi.org/10.33448/rsd-v10i1.12009

\section{Referências}

Federação Brasileira das Associaçoes de Ginecologia e Obstetricia (FEBRASGO). (2017). Pré-eclâmpsia. Série Orientações e Recomendações FEBRASGO. n. 8 .

Ferreira Maria, B. G., Silveira, C. F., Silva, S. R., Souza, D. J., \& Ruiz, M. T. (2016). Assistência de enfermagem a mulheres com pré-eclâmpsia e/ou eclâmpsia: revisão integrativa. Rev. esc. enferm. USP, 50(2), 324-334.

Gil, A. C. (2010). Como Elaborar Projetos de Pesquisa. (5ª ed.): Atlas.

Instituto Brasileiro de Geografia e Estatística (IBGE). Panorama da cidade de São Gabriel na Bahia.

Kerber, G. F., \& Melere, C. (2017). Prevalência de síndromes hipertensivas gestacionais em usuárias de um hospital no sul do Brasil. Rev Cuid., 8(3), 18991906.

Ministério da saúde (BRASIL). (2012). Atenção ao pré-natal de baixo risco. Caderno de Atenção Básica, n. 32, Brasília-DF.

Moura Marta, D. R., Castro, M. D. R., Margotto, P. R., \& Rugolo, L. M. S. S. (2011). Hipertensão Arterial na Gestação - importância do seguimento materno no desfecho neonatal. ComSup. Ciências Saúde, 22(1), 113-119.

Oliveira, A. C. M., \& Graciliano, N. G. (2015). Síndrome hipertensiva da gravidez e diabetes mellitus gestacional em uma maternidade pública de uma capital do Nordeste brasileiro, 2013: prevalência e fatores associados. Epidemiol. Serv. saúde, 24(3), 441-451.

Souza Marilda, G., Lopes, R. G. C., Rocha, M. L. T. L. F., Lippi, U. G., Costa, E. S., \& Santos, C. M. P. (2020). Epidemiology of artherial hypertension in pregnants. Einstein, 18, 1-7.

Thuler Andréa, C. M. C., Wall, M. L., Benedet, D. C. F., Souza, S. R. R. K., \& Souza, M. A. R. (2018). Medidas preventivas das síndromes hipertensivas da gravidez na atenção primária. Rev enferm UFPE on line, Recife, 12(4), 1060-71.

Vasconselos Amorim, T., Souza, I. E. O., Moura, M. A. V., Queiroz, A. B. A., \& Salimena, A. M. O. (2017). Perspectivas do cuidado de enfermagem na gestação de alto risco: revisão integrativa. Rev Enfermeria Global, 46, 515-529. 